\title{
Diabetes and prognosis in older persons with colorectal cancer
}

\author{
J Luo*,1, H-C Lin ${ }^{2}, \mathrm{~K} \mathrm{He}^{1}$ and M Hendryx ${ }^{2}$ \\ ${ }^{1}$ Department of Epidemiology and Biostatistics, School of Public Health - Bloomington, Indiana University, Bloomington, \\ IN, USA and ${ }^{2}$ Department of Applied Health Science, School of Public Health - Bloomington, Indiana University, Bloomington, \\ IN, USA \\ This paper has been modified since Advance Online Publication
}

Background: Epidemiological studies have reported that diabetes significantly increases overall mortality in patients with colorectal cancer. However, it is unclear whether diabetes increases colorectal cancer-specific mortality. We used the US Surveillance Epidemiology and End Results (SEER) database linked with Medicare claims data to assess the influence of pre-existing diabetes on prognosis of patients with colorectal cancer.

Methods: Data from 61213 patients aged 67 or older with colorectal cancer diagnosed between 2003 and 2009 were extracted and prospectively followed through the date of death or the end of 2012 if the patient was still alive. Diabetes cases with and without complications were identified based on an algorithm developed for the Chronic Condition Data Warehouse (CCW). Cox models were used to estimate hazard ratios (HRs) for total mortality. The proportional subdistribution hazards model proposed by Fine and Gray was used to estimate HRs for colorectal cancer-specific mortality.

Results: Compared with patients without diabetes, colorectal cancer patients with pre-existing diabetes had significantly higher risk of overall mortality ( $\mathrm{HR}=1.20,95 \%$ confidence interval $(95 \% \mathrm{Cl}): 1.17-1.23)$. The $\mathrm{HR}$ for overall mortality was more pronounced for patients who had diabetes with complications ( $\mathrm{HR}=1.50,95 \% \mathrm{Cl}$ : 1.42-1.58). However, diabetes was not associated with increased colorectal cancer-specific mortality after accounting for non-colorectal cancer outcomes as competing risk.

Conclusions: Pre-existing diabetes increased risk of total mortality among patients with colorectal cancer, especially among cancer patients who had diabetes with complications. The increased risk of total mortality associated with diabetes was primarily explained by increased cardiovascular-specific mortality, not by increased colorectal cancer-specific mortality.

Colorectal cancer is the fourth most common cancer in the United States, and more than two-thirds of patients diagnosed with colorectal cancer are aged $\geqslant 65$ years (SEER, 2012a, b). Co-morbidity exerts a strong effect on the probability of survival after a cancer diagnosis (Gross et al, 2006; Barone et al, 2008), and there is a critical need for improved understanding of how co-morbid chronic conditions affect outcomes in patients with cancer (Extermann, 2003). Type 2 diabetes is one of the most common chronic diseases (Cowie et al, 2009) and appears to be an independent risk factor for colorectal cancer incidence (Larsson et al, 2005); pre-existing diabetes is present in $\sim 18 \%$ of colorectal cancer cases (Gross et al, 2006). Accumulating epidemiological studies have reported that diabetes significantly increases total mortality in patients with colorectal cancer (Barone et al, 2008; Stein et al, 2010). However, it is unclear whether the higher total mortality in colorectal cancer patients with diabetes is driven by a worse colorectal cancer prognosis or by competing risks such as diabetes-related cardiovascular disease or different clinical practices for cancer patients with pre-existing diabetes.

In the majority of epidemiological applications, competing risk has been ignored (i.e., patients experiencing competing events were censored at the time of these events), which may substantially overestimate the absolute risk of the event of interest and lead to biased findings (Putter et al, 2007; Wolbers et al, 2009). We overcame this limitation through applying an improved analytic approach - proportional subdistribution hazard model proposed

*Correspondence: Dr J Luo; Email: juhluo@indiana.edu

Received 26 November 2013; revised 16 January 2014; accepted 18 January 2014; published online 25 February 2014

(c) 2014 Cancer Research UK. All rights reserved 0007-0920/14 
by Fine and Gray (Fine and Gray, 1999) to estimate colorectal cancer-specific mortality by accounting for non-colorectal cancer outcomes as competing risk.

We used the US Surveillance Epidemiology and End Results (SEER, 2012a,b) database linked with Medicare claims data, a unique population-based source of information, to assess the influence of pre-existing diabetes on prognosis of patients with colorectal cancer. Our study hypothesis was that diabetes would adversely influence colorectal cancer prognosis, including total and cancer-specific mortality. This study aims to address the following questions: (1) Is there a mortality difference between diabetic and non-diabetic patients with colorectal cancer? (2) Is mortality outcome among colorectal cancer patients the result of colorectal cancer prognosis or of risk from non-cancer mortality? (3) Is diabetes associated with unfavourable tumour characteristics? (4) Does the impact of pre-existing diabetes on cancer prognosis differ by patients with and without diabetes complications? Addressing these questions will have great potential to advance the understanding of how pre-existing diabetes influences colorectal cancer prognosis. In particular, we can begin to understand whether diabetes per se may worsen colorectal cancer prognosis, or whether any competing risks may be more important in determining mortality outcomes.

\section{MATERIALS AND METHODS}

Data resource: SEER-Medicare data. The Surveillance, Epidemiology and End Results (SEER, 2012a, b)-Medicare-linked database is used in this project. The SEER programme is an epidemiologic surveillance system sponsored by the US National Cancer Institute (NCI), consisting of population-based tumour registries that routinely collect information on all newly diagnosed cancer cases that occur in persons residing in SEER areas (SEER, $2012 \mathrm{a}, \mathrm{b})$. Since 2000 , the SEER areas capture $\sim 25 \%$ of the US population (SEER, 2012a, b). Cancer registries participating in the SEER programme are required to meet strict standards with respect to case ascertainment and data quality. The information collected about each incident cancer diagnosis includes the patient's demographic characteristics (such as age, sex and race), date of diagnosis, cancer characteristics (e.g., histology, stage and grade), type of surgical treatment and/or radiation therapy recommended or provided within 4 months of diagnosis, followup of vital status and cause of death if applicable (Warren et al, 2002c).

The Medicare programme, federally funded and administered by the Center for Medicare and Medicaid Services (CMS, 2012), provides health insurance for people aged $\geqslant 65$ years, people under age 65 with certain disabilities and people of all ages with end-stage renal disease (permanent kidney failure requiring dialysis or a kidney transplant) (CMS, 2012). Separate claim files can be obtained for inpatient, outpatient, physician and supplier, skilled nursing facility, and hospice services provided to beneficiaries enrolled in fee-for-service plans. Claim files contain diagnosis and procedure codes, dates of services, charges and amount paid.

The SEER-Medicare data reflect the linkage of two large population-based sources of data that provide detailed information about Medicare beneficiaries with cancer. The linkage was first completed in 1991 and has been updated biennially. For each of the linkages, 94 per cent of persons aged $\geqslant 65$ in the SEER files were matched to the Medicare enrolment file; the deficit reflects the 3\% of elderly people who do not enrol in Medicare and another 3\% who do not have sufficient or accurate enough information for the linkage (Engels et al, 2011).

Study population. As of December 2012, the data include all Medicare-eligible persons documented in the SEER data who were diagnosed with cancer through 2009, and their Medicare claims through 2010. Our cohort included patients aged $\geqslant 67$ years in the SEER database who had a first primary diagnosis of invasive colorectal cancers between 2003 and 2009. Sixty-seven years was selected as the age cutoff to ensure that each patient would have at least 2 years of Medicare eligibility before their cancer diagnosis. To ensure a complete assessment of pre-existing diabetes (exposure) and cancer treatment received, we only included patients who were continually enrolled in both Medicare Parts A and $B$ and excluded patients who were enrolled in health maintenance organisation plans over the inclusive 2-year period before colorectal cancer diagnosis and 3 months after cancer diagnosis. Patients in health maintenance organisation plans were excluded because these patients do not have complete claim records. In addition, we excluded patients who had end-stage renal disease or disability alone or who were diagnosed exclusively by death certificates or at autopsy. After considering these inclusion and exclusion criteria, our final study cohort consisted of 61213 patients with colorectal cancer. Of them, 46483 (75.9\%) patients were diagnosed with colon cancer, and 14730 (24.1\%) were diagnosed with rectal cancer.

\section{Measurements}

Outcomes. Our primary outcome is colorectal cancer-specific mortality. However, we also examined total mortality as an outcome for comparison with previous findings.

Pre-existing diabetes status. We adapted an algorithm developed for the Chronic Condition Data Warehouse (CCW Chronic Condition Data Warehouse, 2013) by the Centers for Medicare \& Medicaid Services (CMS, 2012) (CCW Chronic Condition Data Warehouse, 2013) to identify pre-existing diabetes. Diabetes status was determined on the basis of either a single inpatient claim or at least two outpatient claim diagnoses with the International Classification of Disease, 9th Revision, Clinical Modification (ICD-9-CM) diagnosis code of 250.xx during the interval beginning 2 years before and 3 months after colorectal cancer diagnosis. Extending the time interval to 3 months after diagnosis allowed us to capture previously undiagnosed diabetes as other studies have done (Yang et al, 2013). To avoid 'rule out' diagnoses on outpatient claims, a patient's diagnosis must have appeared on at least two different claims that were made $>30$ days apart. We did not include diabetes medications in the definition since Medicare did not begin covering oral medications without an intravenous equivalent until January 2006. We defined diabetes with complication with ICD-9-CM codes 250.4-250.6 or 250.8-250.9 based on the definition of comorbidities described in the National Cancer Institute SEER-Medicare website.

Co-morbidity. Medicare claims were used to calculate the NCI combined co-morbidity index score proposed by Klabunde et al (2007) and as identified by Charlson et al (1987). The NCI index (Klabunde et al, 2000) is composed of two weighted co-morbidity scores derived separately from inpatient and outpatient claims. The NCI combined index uses weights derived from comorbid conditions identified in either Medicare inpatient or outpatient claims into a single co-morbidity index. Study has shown that the new NCI combined index is a more refined, easier to implement co-morbidity measurement algorithm appropriate for investigators using administrative claims databases to study commonly occurring cancers (Klabunde et al, 2007). Two conditions (diabetes without and with complications) pertaining to diabetes were removed from the NCI Co-morbidity Index to reduce correlation with diabetes. ICD-9-CM diagnostic codes recorded in Medicare claims over 2 years before colorectal cancer diagnosis were searched to create this co-morbidity index. Conditions reported 
Table 1. Characteristics of 61213 colorectal cancer patients by diabetes status ${ }^{\mathbf{a}}$

\section{Diabetes}

\begin{tabular}{|l|c|c|c|c|}
\cline { 2 - 5 } & No diabetes & Total no. (\%) & Without complication $^{\text {With complication }}{ }^{\text {b }}$ \\
\hline All patients & 46400 & $14813(24.2)$ & $12298(20.1)$ & $2515(4.1)$ \\
\hline
\end{tabular}

\section{Age (year)}

$65-74$

$75-84$

$85+$

Sex (female, \%)

$16708(36)$

$20744(44)$

$8948(19)$

$25762(56)$

$5965(40)$
$6682(45)$
$2166(15)$
$7785(53)$

4912 (34)

$5556(45)$

1830 (15)

7785 (53)

6501 (53)

1053 (42)

$1126(45)$

\section{Race}

White

Black

American Indian/Alaska Native

Asian or Pacific Islander

Unknown

\begin{tabular}{c|c}
$40650(88)$ & $12167(82)$ \\
$3411(7)$ & $1779(12)$ \\
$115(0)$ & $61(0)$ \\
$2025(4)$ & $754(5)$ \\
$199(0)$ & $52(0)$
\end{tabular}

$10206(83)$
$1383(11)$
$47(0)$
$616(5)$
$46(0)$

$1961(78)$

$396(16)$

$199(0)$

$46(0)$

$138(6)$

$<11$ ( )

\section{Marital status}

\begin{tabular}{|l|}
\hline Single (never married) \\
Married \\
Separated \\
Divorced \\
Widowed \\
Unknown
\end{tabular}

$3623(8)$

22715 (49)

270 (1)

$2881(6)$

14881 (32)

2030 (4)

\section{Median Income}

Lowest quartile

Second quartile

Third quartile

Highest quartile

$11092(24)$

$11483(25)$

$11666(25)$

12159 (26)

$1169(8)$
$7151(48)$
$121(1)$
$1003(7)$
$4711(32)$
$658(4)$

$954(8)$

6010 (49)

101 (1)

807 (7)

3871 (32)

555 (6)

$215((9)$

1141 (45)

20 (1)

$196(8)$

840 (33)

103 (4)

\section{No. of comorbidities}

\begin{tabular}{|l|c|r|}
\hline 0 & $37348(81)$ & $9624(65)$ \\
1 & $5323(12)$ & $2313(16)$ \\
\hline
\end{tabular}

$\geqslant 2$

Cancer site at diagnosis

\begin{tabular}{|c|c|c|c|c|}
\hline $\begin{array}{l}\text { Colon } \\
\text { Rectum }\end{array}$ & $\begin{array}{r}34851(75) \\
11549(25)\end{array}$ & $\begin{array}{r}11632(79) \\
3181(21)\end{array}$ & $\begin{array}{l}9598(78) \\
2700(22)\end{array}$ & $\begin{array}{r}2034(81) \\
481(19)\end{array}$ \\
\hline \multicolumn{5}{|l|}{ Cancer stage } \\
\hline $\begin{array}{l}\text { Localised } \\
\text { Regional } \\
\text { Distant } \\
\text { Unknown }\end{array}$ & $\begin{array}{c}20088(43) \\
17520(38) \\
6679(14) \\
2113(5)\end{array}$ & $\begin{array}{c}6478(44) \\
5758(39) \\
1921(13) \\
656(4)\end{array}$ & $\begin{array}{c}5336(43) \\
4822(39) \\
1617(13) \\
523(4)\end{array}$ & $\begin{array}{c}1142(45) \\
936(37) \\
304(12) \\
133(5)\end{array}$ \\
\hline \multicolumn{5}{|l|}{ Grade } \\
\hline $\begin{array}{l}\text { Grade I: well differentiated } \\
\text { Grade II: moderately differentiated } \\
\text { Grade III: poorly differentiated } \\
\text { Grade IV: undifferentiated } \\
\text { Unknown }\end{array}$ & $\begin{array}{r}4132(9) \\
28262(61) \\
7762(17) \\
659(1) \\
5585(12)\end{array}$ & $\begin{array}{c}1335(9) \\
9258(63) \\
2403(16) \\
205(1) \\
1612(11)\end{array}$ & $\begin{aligned} 1097(9) \\
7723(63) \\
1981(16) \\
171(1) \\
1326(11)\end{aligned}$ & $\begin{aligned} 238(10) \\
1535(61) \\
422(17) \\
34(1) \\
286(11)\end{aligned}$ \\
\hline \multicolumn{5}{|l|}{ Cancer-direct surgery } \\
\hline $\begin{array}{l}\text { No } \\
\text { Yes } \\
\text { Unknown }\end{array}$ & $\begin{array}{r}5099(11) \\
40915(88) \\
386(1)\end{array}$ & $\begin{array}{r}1533(10) \\
13158(89) \\
122(1)\end{array}$ & $\begin{array}{r}1237(10) \\
10967(89) \\
94(1)\end{array}$ & $\begin{array}{r}296(12) \\
2191(87) \\
28(1)\end{array}$ \\
\hline \multicolumn{5}{|l|}{ Radiation therapy } \\
\hline $\begin{array}{l}\text { No } \\
\text { Yes } \\
\text { Unknown }\end{array}$ & $\begin{array}{c}41187(89) \\
4628(10) \\
585(1)\end{array}$ & $\begin{array}{r}13395(9) \\
1214(8) \\
204(1)\end{array}$ & $\begin{array}{c}11089(90) \\
1043(9) \\
166(1)\end{array}$ & $\begin{array}{c}2306(92) \\
171(7) \\
38(2)\end{array}$ \\
\hline \multicolumn{5}{|l|}{ Chemotherapy } \\
\hline Yes & $4055(9)$ & $1338(9)$ & $1161(9)$ & $177(7)$ \\
\hline
\end{tabular}


within 1 month of cancer diagnosis were excluded to avoid misclassifying complications or conditions directly resulting from cancer diagnosis or treatment as co-morbidities (Klabunde et al, 2007).

Cancer stage and tumour characteristics. The colorectal cancer information was extracted from SEER data. The cancer stage was categorised as localised (confined to primary site), regional (spread to regional lymph nodes), distant (cancer has metastasised) or unknown (unstaged). Other tumour characteristics included tumour grade (grade I - well differentiated; grade II - moderately differentiated; grade III - poorly differentiated and grade IV undifferentiated), and different histological subtypes of colorectal cancer (colon or rectum).

Cancer treatment. The SEER programme routinely collects information regarding certain anti-cancer therapies (i.e. surgery, radiation therapy) occurring within 4 months of diagnosis (first course of therapy). For surgery, we divided patients as two categories: cancer-directed surgery performed or not. For the method of radiation therapy performed as part of the first course of treatment, we collapsed patients who received any radiation (such as bean radiation, radioactive implants, radioisotopes or combination) as yes for radiation. As SEER does not report information pertaining to chemotherapy administration, we searched claims records to identify chemotherapy. Patients who had at least one claims record for chemotherapeutic administration, treatment or agents in any of inpatient and outpatient claims files within 6 months after primary diagnosis were considered chemotherapy recipients. We used codes including ICD-9-CM diagnosis codes (V58.1, V66.2 and V67.2), ICD-9-CM procedure code (99.25) and HCPCS codes (964xx, 965xx, Q0083-Q0085, J9XXX, J8510, J852x, J8530, J856x, J8600, J8610, J870x and J8999) (Warren et al, 2002a; Yang et al, 2013).

Covariates. In the multivariate model, we adjusted for demographic variables including patient's demographic characteristics (age at diagnosis, sex, race and marital status), and socioeconomic status (median household income). The median income in each patient's census tract was used as a proxy measure for socioeconomic status. It was estimated at census tract level using the 2000 census and stratified into quartiles. Race was categorised as white, black, American Indian/Alaska Native, Asian or Pacific Islander and others.

Supplementary Table 1 shows all of the ICD-9 codes listed in the paper.

Statistical analysis. Distribution of baseline patients' characteristics, tumour characteristics and stage at diagnosis were compared between patients with and without diabetes. $\chi^{2}$-tests were used to evaluate differences for categorical covariates, and $t$-tests were used for continuous variables. Age-adjusted and multivariate-adjusted Cox proportional hazards models were used to estimate adjusted hazard ratios (HRs) and 95\% confidence intervals (95\% CI) for overall survival. The proportional subdistribution hazard model proposed by Fine and Gray (1999) was used to estimate HRs for colorectal cancer-specific mortality associated with diabetes status by accounting for non-colorectal cancer outcomes as competing risk. In the multivariate models, we adjusted for covariates including age at diagnosis $(67-69,70-74,75-79,80-84$ and $85+$ ), sex, race (white, black, American Indian/Alaska Native, Asian or Pacific Islander, other), marital status (never married, married, separated, divorced and widowed), grade (grade I - well differentiated; grade II - moderately differentiated; grade III poorly differentiated and grade IV - undifferentiated), census tract median income (quartiles) and co-morbidity $(0,1,2+)$.
The underlying time metric in the Cox model was follow-up time since diagnosis of cancer to the date of death or the end of 2012 if the patient was still alive. The date of death from any cause was used for total mortality; the date of death from colorectal cancer was used for cancer-specific mortality for colorectal, colon and rectal cancer patients. The proportionality assumption was confirmed for all exposure variables of interest and for all potential confounding variables, based on graphs of scaled Schoenfeld residuals (Hess, 1995).

\section{RESULTS}

Of a total of 61213 colorectal cancer patients, 14813 (24.2\%) had diabetes including 12298 without complications and 2515 with complications. Over an average of 38 months of follow-up (median = 33 months, range 0-96 months), 28682 (46.9\%) patients died from all causes, and 15879 (25.9\%) patients died from colorectal cancer.

Baseline patients' characteristics by diabetes status are shown in Table 1. Compared with patients without diabetes, patients with diabetes were significantly younger, males, members of non-White race groups, unmarried and from low-median income areas.
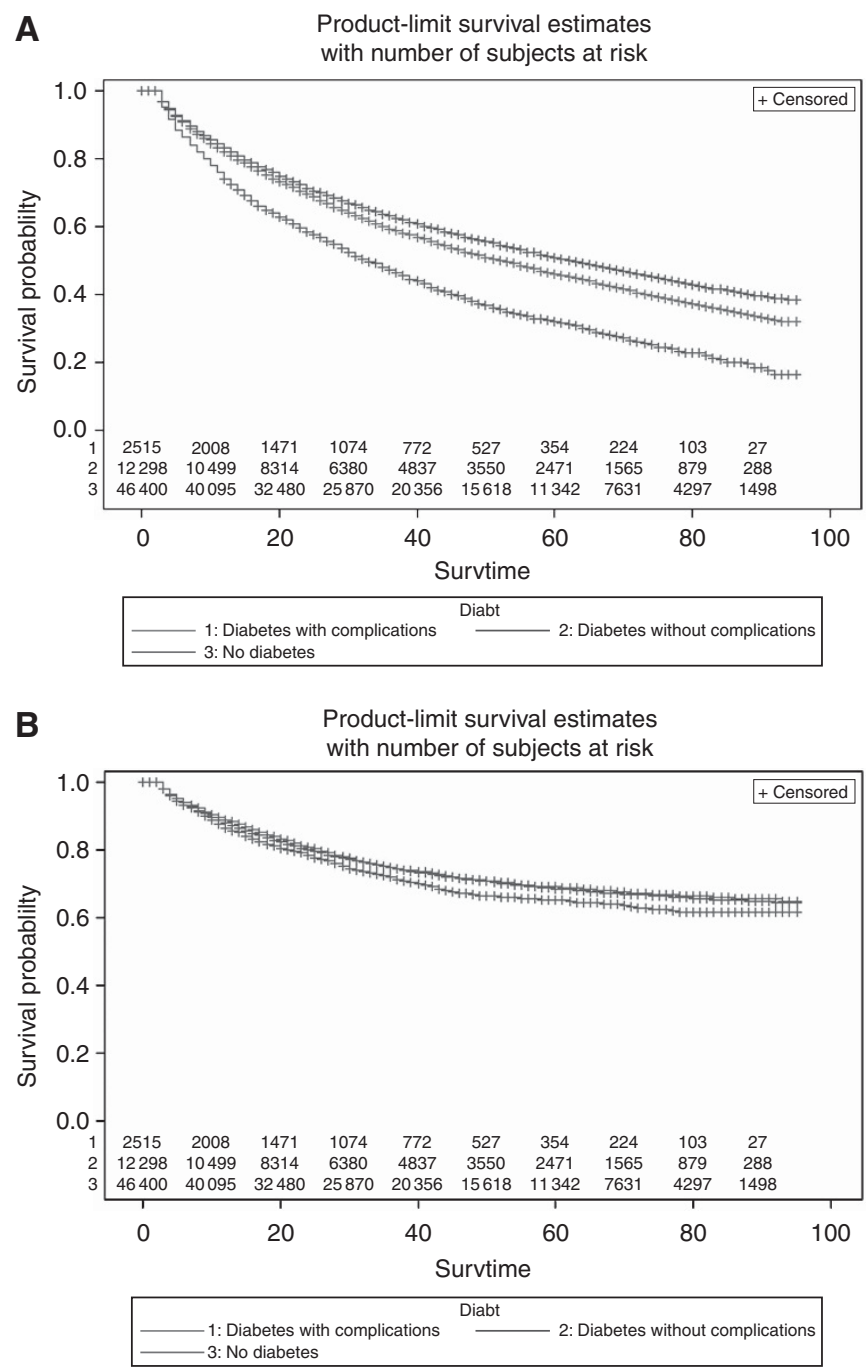

Figure 1. Survival curves among colorectal cancer patients by diabetes status (A) for total survival rates (log-rank test $P$-value $<0.0001$ ); (B) colorectal-cancer-specific survival rates (log-rank test $P$-value $=0.001$ ). 
Patients with diabetes were also more likely to have one or more co-morbidities, and were less likely to have radiation therapy performed as part of the first course of cancer treatment. There was no substantial difference between the diabetes group and the nondiabetes group in terms of the stage of diagnosis, tumour grade and whether the patient underwent cancer-direct surgery or chemotherapy, although $P$-values for statistical tests were significant due to the large sample size. The patterns were similar when comparing patients with complication to patients without complication among patients with diabetes (Table 1).

Figure 1 shows the crude total and colorectal cancer-specific survival curves by diabetes status. Diabetes with complications had the lowest total survival rates and the lowest colorectal cancerspecific mortality. There was no notable difference in colorectal cancer-specific survival rates between patients without diabetes and patients with diabetes but no complications.

Compared with patients without diabetes, we observed that colorectal cancer patients with pre-existing diabetes had significantly higher risk of total mortality $(\mathrm{HR}=1.20,95 \% \mathrm{CI}: 1.17-1.23$ for patients in all stages, $\mathrm{HR}=1.25,95 \% \mathrm{CI}: 1.21-1.29$ for patients in localised or regional stage and $\mathrm{HR}=1.12$, $95 \mathrm{CI}$ : $1.06-1.18$ for patients in distant stage) after adjusting for potential confounders. The risks of total mortality were more pronounced for patients who had diabetes with complications $(\mathrm{HR}=1.50,95 \% \mathrm{CI}: 1.42-1.58$ for patients in all stages, $\mathrm{HR}=1.63,95 \% \mathrm{CI}$ : $1.53-1.73$ for patients in localised or regional stage and $\mathrm{HR}=1.21,95 \% \mathrm{CI}$ : $1.07-1.37$ for patients in distant). Similar findings were observed when we separated colon and rectal cancer patients with an exception that the results for distant-stage rectal cancer patients become non-significant (Table 2). We observed that diabetes was significantly associated with cardiovascular-specific mortality regardless of the site of cancer, especially for diabetes with complications ( $\mathrm{HR}=2.27,95 \% \mathrm{CI}$ : 2.06-2.50) (Table 2).

In contrast, we did not observe a significantly increased risk for colorectal-specific mortality among patients with colon, rectal or colorectal cancer regardless of stage and diabetes severity (Table 3 ). There was one exception, as diabetes with complications and advanced-stage colon cancer was significantly associated with colorectal cancer mortality.

Finally, we performed analyses stratified by sex for colorectalspecific mortality associated with diabetes. No significant difference was found between females and males (data not shown).

\section{DISCUSSION}

The present study found that colorectal cancer patients with preexisting diabetes had significantly higher risk of total mortality than those cancer patients without diabetes. The risk was more pronounced among those who had diabetes with complications. Further performing specific mortality analyses using the competing risk method, diabetes was significantly associated with cardiovascular-specific mortality, but not with colorectal cancer-specific mortality.

Our findings were in agreement with the majority of the literature in term of total mortality as an outcome (Barone et al, 2008; Stein et al, 2010; Huang et al, 2011; Dehal et al, 2012; van de Poll-Franse et al, 2012; Bella et al, 2013; Jeon et al, 2013;

Table 2. Effect of pre-existing diabetes on total mortality in patients with colorectal cancer, by stage

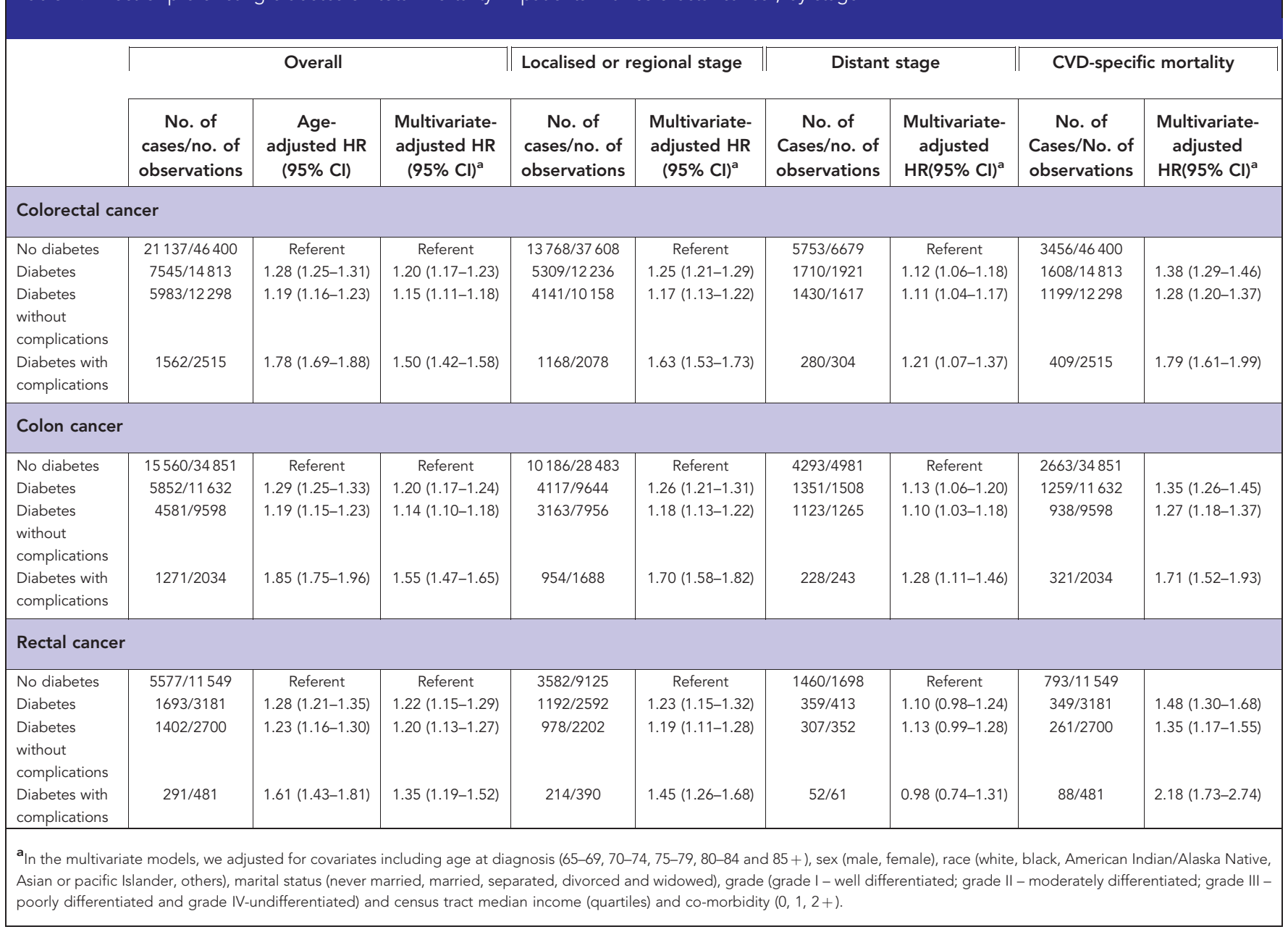


Table 3. Effect of pre-existing diabetes on cancer-specific mortality in patients with colorectal cancer, by stage

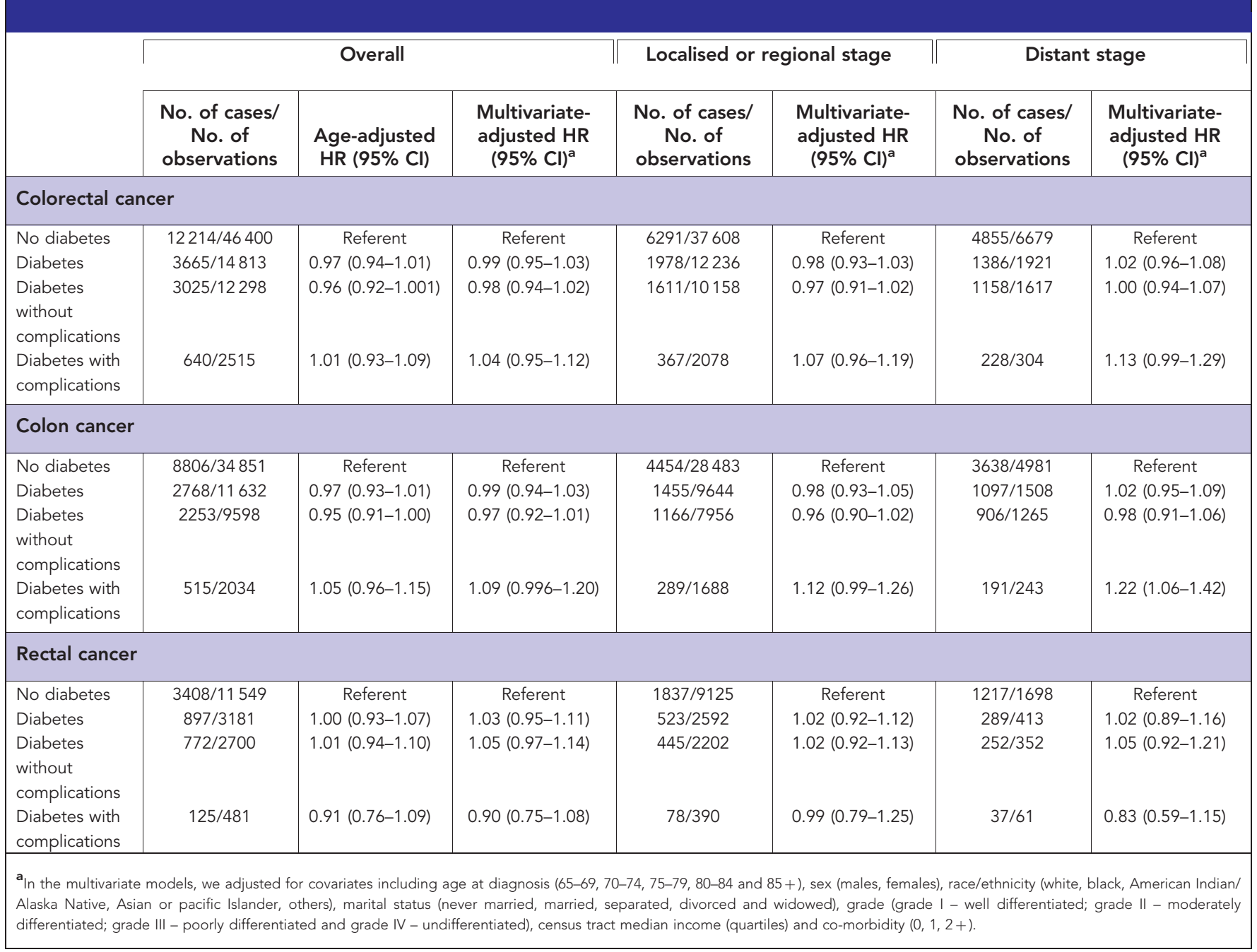

Walker et al, 2013; Yang et al, 2013), although not all studies have found this relationship (Jullumstro et al, 2009; Call et al, 2010; Chen et al, 2010; Noh et al, 2010; Huang et al, 2012). Subgroup analyses of two meta-analysis studies (Barone et al, 2008; Stein et al, 2010) based on six studies showed that colorectal cancer patients with diabetes had $32 \%$ increased risk of total mortality compared with those without diabetes (95\% CI: 1.24-1.41). Among studies (Will et al, 1998; Polednak, 2006; Siddiqui et al, 2008; Jullumstro et al, 2009; Huang et al, 2011, 2012; van de Poll-Franse et al, 2012; Bella et al, 2013; Cossor et al, 2013; Walker et al, 2013) that examined cancer-specific mortality associated with preexisting diabetes, the findings are inconsistent. Of them, one study (Huang et al, 2011) found a significant increased risk for colon cancer-specific mortality. Two (van de Poll-Franse et al, 2012; Bella et al, 2013) found a significantly increased risk for only rectal cancer patients but not for colon cancer, and one (Siddiqui et al, 2008) found an association between poorly controlled pre-existing diabetes and the risk of death attributed to colorectal cancer. Other studies found no significant association between diabetes and subsequent death from colorectal cancer. In addition, an earlier study on this topic (Meyerhardt et al, 2003) showed diabetes had worse disease-free survival associated with diabetes.

However, among all previous studies examining cancer-specific mortality, none of them considered competing risk correctly; rather, they censored patients experiencing competing events at the time of these events, which may substantially overestimate the absolute risk of the event of interest (Putter et al, 2007; Wolbers et al, 2009). For the purpose of comparison with previous studies, we used conventional epidemiology methods to analyse colorectalcancer-specific mortality; the resulting HRs were 1.05 (95\% CI: 1.01-1.09) and 1.17 (95\% CI: 1.08-1.27) for colorectal cancerspecific mortality associated with pre-existing diabetes without and with complications, respectively. Comparing the two analytic approaches, our analyses suggest that findings for cancer-specific mortality using conventional epidemiological methods were overestimated.

The potential influence of diabetes on cancer prognosis is complex. Diabetes may directly influence cancer progression and outcome via physiologic effects of hyperinsulinemia and/or hyperglycaemia (Richardson and Pollack, 2005; Morss and Edelman, 2007). Although our data did not directly assess the association between insulin and colorectal cancer prognosis, experimental and epidemiological evidence suggests that hyperinsulinemia may be an underlying mechanism to explain the association between diabetes and cancer incidence and outcome (Larsson et al, 2005; Berster and Goke, 2008; Giovannucci et al, 2010). Second, pre-existing diabetes may also have indirect adverse effects on cancer outcome by influencing patients or providers to make different clinical decisions regarding cancer screening and cancer treatment. Research has documented underuse of colorectal cancer screening among elderly diabetic women compared with those without diabetes (McBean and Yu, 2007), which may lead to 
detection at a later stage on diagnosis. There may also be decisions to follow less-aggressive cancer treatments among diabetes patients (van de Poll-Franse et al, 2007). However, our data show that there were no substantial differences between the diabetes group and the non-diabetes group in terms of the stage of diagnosis, tumour grade or whether the patient underwent cancer-direct surgery or chemotherapy, although radiation therapy performed as part of the first course of treatment was slightly lower in diabetes patients.

Our study using competing risk methods observed that diabetes was associated with cardiovascular-specific mortality, but not with colorectal-cancer-specific mortality. The findings indicate that diabetes per se may not worsen colorectal cancer prognosis, and that other competing risks such as cardiovascular diseases may be more important in determining mortality outcomes. Thus, besides cancer treatment, preventing patients from developing diabetes and having proper management of diabetes for diabetic patients are also important in improving prognosis for patients with colorectal cancer.

The strengths of the present study include using a large, US nationally representative database, the availability of detailed clinical information on cancer and some data regarding severity of diabetes status. The SEER-Medicare data are a unique resource that combines clinical information from population-based cancer registries with claims information from the Medicare programme. Extraction of all of the Medicare claims for each cancer patient makes it possible to longitudinally track persons from their Medicare eligibility until death.

There are several limitations in the present study. First, our study typically relied on existing public health surveillance and administrative information that were not designed for this research purpose. Challenges in utilising existing data include lack of other information; for example, some demographic and lifestyle variables such as patients' income, BMI, smoking and alcohol habits are missing. As BMI, smoking or other lifestyle variables may influence survival after a diagnosis of colorectal cancer, if our diabetic colorectal cancer patients were more likely to have high BMI or to have smoked, the worse total survival independently associated with diabetes may be overestimated. Another concern is the completeness and accuracy of Medicare claims. To increase accuracy, we used an algorithm to identify conditions that required two outpatient claims or one in-patient claim. A previous study reported that this algorithm with Medicare claims data identified $69 \%$ of pre-existing diabetes cases (Gorina and Kramarow, 2011). The claims-based algorithm we used to identify diabetes has a validated sensitivity of $74.4 \%$ and specificity of $97.5 \%$ using a 2-year look-back period (Hebert et al, 1998). Thus, we may have missed some cases of diabetes. In addition, the restricted window available in claims data may be a concern. Specifically, claims data are not available before the age of 65 years, which limits our analysis to patients who are older than 67 years; thus, our findings may only be generalised to older patients enrolled in non-HMO Medicare, although colorectal cancer occurs disproportionately in the elderly with more than two-thirds of all cases occurring in persons aged $\geqslant 65$ years (SEER, 2012a,b); further, studies have shown that age, sex and other sociodemographic features of the elderly SEER population are comparable with that of the US elderly population (Warren et al, 2002b). Moreover, we had no information on medication use, because our study cohort predated the advent of Medicare Part D. Metformin, an oral drug widely used as a first-line therapy for type 2 diabetes, has been associated with a lower risk of colorectal incidence compared with other antidiabetic therapies, such as insulin and sulfonylureas (Zhang et al, 2011). Studies have also shown that metformin use may lower risk of colorectal cancer-specific and total mortality (Lee et al, 2012). However, a recent study reported that the use of metformin was not associated with the incidence of colorectal cancer (Smiechowski et al, 2013).
In conclusion, our large population-based study provides additional evidence that pre-existing diabetes increased risk of total mortality among colorectal cancer patients. The increased total mortality associated with diabetes was mainly driven by increased risk of dying from cardiovascular diseases. Preventing diabetes and reducing diabetes complications may improve the survival rate of colorectal cancer patients.

\section{ADDENDUM}

To comply with SEER-Medicare data rules on confidentiality, data in the 'Diabetes/With complication (Race)' cell in Table 1 has been updated since Advance Online Publication and a footnote added to the table.

\section{REFERENCES}

Barone BB, Yeh HC, Snyder CF, Peairs KS, Stein KB, Derr RL, Wolff AC, Brancati FL (2008) Long-term all-cause mortality in cancer patients with preexisting diabetes mellitus: a systematic review and meta-analysis. JAMA 300(23): 2754-2764.

Bella F, Minicozzi P, Giacomin A, Crocetti E, Federico M, de Leon MP, Fusco M, Tumino R, Mangone L, Giuliani O, Budroni M, Sant M (2013) Impact of diabetes on overall and cancer-specific mortality in colorectal cancer patients. J Cancer Res Clin Oncol 139(8): 1303-1310.

Berster JM, Goke B (2008) Type 2 diabetes mellitus as risk factor for colorectal cancer. Arch Physiol Biochem 114(1): 84-98.

Call R, Grimsley M, Cadwallader L, Cialone L, Hill M, Hreish V, King ST, Riche DM (2010) Insulin - carcinogen or mitogen? Preclinical and clinical evidence from prostate, breast, pancreatic, and colorectal cancer research. Postgrad Med 122(3): 158-165.

CCW Chronic Condition Data Warehouse (2013) https://www.ccwdata.org/ web/guest/condition-categories.

Charlson ME, Pompei P, Ales KL, MacKenzie CR (1987) A new method of classifying prognostic comorbidity in longitudinal studies: development and validation. J Chronic Dis 40(5): 373-383.

Chen CQ, Fang LK, Cai SR, Ma JP, Yang GX, Yang W, Zhan WH, He YL (2010) Effects of diabetes mellitus on prognosis of the patients with colorectal cancer undergoing resection: a cohort study with 945 patients. Chinese Med J-Peking 123(21): 3084-3088.

CMS (2012) http://www.cms.gov/MedicareGenInfo/Vol.

Cossor FI, Adams-Campbell LL, Chlebowski RT, Gunter MJ, Johnson K, Martell RE, McTiernan A, Simon MS, Rohan T, Wallace RB, Paulus JK (2013) Diabetes, metformin use, and colorectal cancer survival in postmenopausal women. Cancer Epidemiol 37(5): 742-749.

Cowie CC, Rust KF, Ford ES, Eberhardt MS, Byrd-Holt DD, Li C, Williams DE, Gregg EW, Bainbridge KE, Saydah SH, Geiss LS (2009) Full accounting of diabetes and pre-diabetes in the U.S. population in 1988-1994 and 2005-2006. Diabetes Care 32(2): 287-294.

Dehal AN, Newton CC, Jacobs EJ, Patel AV, Gapstur SM, Campbell PT (2012) Impact of diabetes mellitus and insulin use on survival after colorectal cancer diagnosis: the Cancer Prevention Study-II Nutrition Cohort. J Clin Oncol 30(1): 53-59.

Engels EA, Pfeiffer RM, Ricker W, Wheeler W, Parsons R, Warren JL (2011) Use of surveillance, epidemiology, and end results-medicare data to conduct case-control studies of cancer among the US elderly. Am J Epidemiol 174(7): 860-870.

Extermann M (2003) Studies of comprehensive geriatric assessment in patients with cancer. Cancer Control 10(6): 463-468.

Fine JP, Grey RJ (1999) A proportional hazards model for the subdistribution of a competing risk. J Am Stat Assoc 94(446): 496-509.

Giovannucci E, Harlan DM, Archer MC, Bergenstal RM, Gapstur SM, Habel LA, Pollak M, Regensteiner JG, Yee D (2010) Diabetes and cancer: a consensus report. Diabetes Care 33(7): 1674-1685.

Gorina Y, Kramarow EA (2011) Identifying chronic conditions in Medicare claims data: evaluating the Chronic Condition Data Warehouse algorithm. Health Services Res 46(5): 1610-1627. 
Gross CP, Guo Z, McAvay GJ, Allore HG, Young M, Tinetti ME (2006) Multimorbidity and survival in older persons with colorectal cancer. J Am Geriatr Soc 54(12): 1898-1904.

Hebert PL, Engelgau MM, Geiss LS, Tierney E, Riley G, Mcbean AM (1998) Identifying diabetes in the Medicare population using Medicare claims data. Diabetes 47: A187-A187.

Hess KR (1995) Graphical methods for assessing violations of the proportional hazards assumption in Cox regression. Stat Med 14(15): 1707-1723.

Huang CW, Sun LC, Shih YL, Tsai HL, Chen CW, Yeh YS, Ma CJ, Huang CJ, Wang JY (2012) The impact on clinical outcome of high prevalence of diabetes mellitus in Taiwanese patients with colorectal cancer. World J Surg Oncol 10: 76.

Huang YC, Lin JK, Chen WS, Lin TC, Yang SH, Jiang JK, Chang SC, Lan YT, Wang HS, Liu CY, Yang YW, Teng HW (2011) Diabetes mellitus negatively impacts survival of patients with colon cancer, particularly in stage II disease. J Cancer Res Clin Oncol 137(2): 211-220.

Jeon JY, Jeong DH, Park MG, Lee JW, Chu SH, Park JH, Lee MK, Sato K, Ligibel JA, Meyerhardt JA, Kim NK (2013) Impact of diabetes on oncologic outcome of colorectal cancer patients: colon $v s$ rectal cancer. Plos One 8(2): e55196.

Jullumstro E, Kollind M, Lydersen S, Edna TH (2009) Diabetes mellitus and outcomes of colorectal cancer. Acta Oncol 48(3): 361-367.

Klabunde CN, Legler JM, Warren JL, Baldwin LM, Schrag D (2007) A refined comorbidity measurement algorithm for claims-based studies of breast, prostate, colorectal, and lung cancer patients. Ann Epidemiol 17(8): 584-590.

Klabunde CN, Potosky AL, Legler JM, Warren JL (2000) Development of a comorbidity index using physician claims data. J Clin Epidemiol 53(12): $1258-1267$.

Larsson SC, Orsini N, Wolk A (2005) Diabetes mellitus and risk of colorectal cancer: a meta-analysis. J Natl Cancer Inst 97(22): 1679-1687.

Lee JH, Kim TI, Jeon SM, Hong SP, Cheon JH, Kim WH (2012) The effects of metformin on the survival of colorectal cancer patients with diabetes mellitus. Int J Cancer 131(3): 752-759.

McBean AM, Yu XH (2007) The underuse of screening services among elderly women with diabetes. Diabetes Care 30(6): 1466-1472.

Meyerhardt JA, Catalano PJ, Haller DG, Mayer RJ, Macdonald JS, Benson 3rd AB, Fuchs CS (2003) Impact of diabetes mellitus on outcomes in patients with colon cancer. J Clin Oncol 21(3): 433-440.

Morss AS, Edelman ER (2007) Glucose modulates basement membrane fibroblast growth factor-2 via alterations in endothelial cell permeability. J Biol Chem 282(19): 14635-14644.

Noh GY, Hwang DY, Choi YH, Lee YY (2010) Effect of diabetes mellitus on outcomes of colorectal cancer. J Korean Soc Coloproctol 26(6): 424-428.

Polednak AP (2006) Comorbid diabetes mellitus and risk of death after diagnosis of colorectal cancer: a population-based study. Cancer Detect Prevent 30(5): 466-472.

Putter H, Fiocco M, Geskus RB (2007) Tutorial in biostatistics: competing risks and multi-state models. Stat Med 26(11): 2389-2430.

Richardson LC, Pollack LA (2005) Therapy insight: influence of type 2 diabetes on the development, treatment and outcomes of cancer. Nat Clin Pract Oncol 2(1): 48-53.

SEER (2012a) http://seer.cancer.gov/about/.
SEER (2012b) http://seer.cancer.gov/statfacts/html/colorect.html.

Siddiqui AA, Spechler SJ, Huerta S, Dredar S, Little BB, Cryer B (2008) Elevated $\mathrm{HbAlc}$ is an independent predictor of aggressive clinical behavior in patients with colorectal cancer: A case-control study. Digest Dis Sci 53(9): 2486-2494.

Smiechowski B, Azoulay L, Yin H, Pollak MN, Suissa S (2013) The use of metformin and colorectal cancer incidence in patients with type II diabetes mellitus. Cancer Epidemiol Biomarkers Prevent 22(10): 1877-1883.

Stein KB, Snyder CF, Barone BB, Yeh HC, Peairs KS, Derr RL, Wolff AC, Brancati FL (2010) Colorectal cancer outcomes, recurrence, and complications in persons with and without diabetes mellitus: a systematic review and meta-analysis. Dig Dis Sci 55(7): 1839-1851.

van de Poll-Franse LV, Haak HR, Coebergh JWW, Janssen-Heijnen MLG, Lemmens VEPP (2012) Disease-specific mortality among stage I-III colorectal cancer patients with diabetes: a large population-based analysis. Diabetologia 55(8): 2163-2172.

van de Poll-Franse LV, Houterman S, Janssen-Heijnen MLG, Dercksen MW, Coebergh JWW, Haak HR (2007) Less aggressive treatment and worse overall survival in cancer patients with diabetes: a large population based analysis. Int J Cancer 120(9): 1986-1992.

Walker JJ, Brewster DH, Colhoun HM, Fischbacher CM, Lindsay RS, Wild SH. SDRN SDRN (2013) Cause-specific mortality in Scottish patients with colorectal cancer with and without type 2 diabetes (2000-2007). Diabetologia 56(7): 1531-1541.

Warren JL, Harlan LC, Fahey A, Virnig BA, Freeman JL, Klabunde CN, Cooper GS, Knopf KB (2002a) Utility of the SEER-Medicare data to identify chemotherapy use. Med Care 40(8): 55-61.

Warren JL, Klabunde CN, Schrag D, Bach PB, Riley GF (2002b) Overview of the SEER-Medicare data-content, research applications, and generalizability to the United States elderly population. Med Care 40(8): 3-18.

Warren JL, Klabunde CN, Schrag D, Bach PB, Riley GF (2002c) Overview of the SEER-Medicare data: content, research applications, and generalizability to the United States elderly population. Med Care 40(8 Suppl): IV-3-18.

Will JC, Galuska DA, Vinicor F, Calle EE (1998) Colorectal cancer: another complication of diabetes mellitus? Ame J Epidemiol 147(9): 816-825.

Wolbers M, Koller MT, Witteman JC, Steyerberg EW (2009) Prognostic models with competing risks: methods and application to coronary risk prediction. Epidemiology 20(4): 555-561.

Yang Y, Mauldin PD, Ebeling M, Hulsey TC, Liu B, Thomas MB, Camp ER, Esnaola NF (2013) Effect of metabolic syndrome and its components on recurrence and survival in colon cancer patients. Cancer 119(8): 1512-1520.

Zhang ZJ, Zheng ZJ, Kan H, Song Y, Cui W, Zhao G, Kip KE (2011) Reduced risk of colorectal cancer with metformin therapy in patients with type 2 diabetes: a meta-analysis. Diabetes Care 34(10): 2323-2328.

This work is published under the standard license to publish agreement. After 12 months the work will become freely available and the license terms will switch to a Creative Commons AttributionNonCommercial-Share Alike 3.0 Unported License.

Supplementary Information accompanies this paper on British Journal of Cancer website (http://www.nature.com/bjc) 